\title{
Association between metabolic syndrome and blepharoptosis in the Korean adults: the Korea National Health and Nutrition Examination Survey 2010-2012
}

\author{
Changhyun Koh, MD¹, Geunyeong Kim, MD¹, Kyungdo Han, $\mathrm{MS}^{2}$, Juwan Park, MD, PhD ${ }^{1}$ \\ ${ }^{1}$ Department of Ophthalmology, Yeouido St. Mary's Hospital, College of Medicine, The Catholic University of Korea, Seoul, Rep. of Korea, \\ ${ }^{2}$ Department of Medical Statistics, College of Medicine, The Catholic University of Korea, Seoul, Rep. of Korea
}

\begin{abstract}
Background: Metabolic syndrome (MetS) is a complex disorder featuring chronic inflammation characterized by abdominal obesity, hyperglycemia, hypertension, and dyslipidemia. Recent studies have documented that MetS is associated with various diseases, including ocular disorders.

Objective: This cross-sectional study is based on data from the fifth Korea National Health and Nutrition Examination Surveys. We examined the association between MetS and its components with blepharoptosis in Korean adults aged 19 years or older.

Methods: MetS was defined using the criteria proposed by the American Heart Association and the National Heart, Lung, and Blood Institute together with the International Diabetes Federation in 2009. Blepharoptosis was defined as the marginal reflex distance 1 of $<2 \mathrm{~mm}$.

Results: The prevalence of blepharoptosis was $11.5 \%$. As the number of MetS components increased, the prevalence of blepharoptosis also increased. After being controlled for various confounders, MetS was significantly associated with blepharoptosis (adjusted odds ratio [OR], 1.316; 95\% confidence interval [CI], 1.133-1.529). In addition, blepharoptosis was associated with increased waist circumference, hypertension, and elevated triglyceride (adjusted OR, 95\% Cl; 1.281 [1.047-1.567], 1.165 [1.014-1.339], and 1.174 [1.013-1.36], respectively).

Conclusion: Consequently, MetS and its components were associated with blepharoptosis in Korean adults. Screening for blepharoptosis is warranted for patients with MetS.
\end{abstract}

Keywords: blepharoptosis; metabolic syndome

\section{Introduction}

Blepharoptosis is defined as an abnormally low-lying upper eyelid margin in primary gaze [1]. Etiologically, blepharoptosis may be due to myogenic, neurogenic, aponeurotic, mechanical, or traumatic causes [1-3]. It is a frequent condition among oculoplastic patients, and its clinical picture varies from a mi- nor cosmetic discomfort to severe visual dysfunction, depending on the pathogenesis and the degree of blepharoptosis [4]. Blepharoptosis is usually an isolated disease, which changes the patient's appearance, but may be associated with various conditions, such as immunological, degenerative, or congenital disorders, tumor, and vascular disease, and may be one of the early presenting symptoms of an underlying disease [1]. Blepharop-

Received August 30, 2018; Revised November 28, 2018; Accepted December 7, 2018

Corresponding author: Juwan Park

E-mail: parkjw@catholic.ac.kr, ORCID: https://orcid.org/0000-0002-5997-8746

This is an Open Access article distributed under the terms of the Creative Commons Attribution Non-Commercial License (http://creativecommons.org/licenses/by-nc/4.0), which permits unrestricted non-commercial use, distribution, and reproduction in any medium, provided the original work is properly cited.

Copyright (c) 2018 Korean Society of Korean Cosmetic Surgery and Medicine (KSKCS \& KCCS). 
tosis may be caused by weakness of the levator palpebrae superioris or may damage nerves that control the muscle, resulting from diabetes. Diabetes and insulin-resistant state, such as impaired glucose tolerance, are also known to be associated with blepharoptosis $[5,6]$. The treatment of blepharoptosis depends on the underlying etiology. Successful treatment of underlying medical problems may sometimes correct blepharoptosis. Thus, early diagnosis and treatment are important prognostic factors in its management.

Metabolic syndrome (MetS) is a complex disorder featuring chronic inflammation characterized by abdominal obesity, hyperglycemia, hypertension, and dyslipidemia [7]. Its definition varies by the organization and expert group. MetS was defined based on the Joint Interim Statement proposed in 2009 from the International Diabetes Federation (IDF) and American Heart Association (AHA)/National Heart, Lung, and Blood Institute (NHLBI) [8]. A decade of small epidemiology studies provided evidence that early hyperglycemia or MetS is sufficient to damage the distal peripheral nerves [9-11]. Obesity was found to be one of the possible factors that cause blepharoptosis [12]. Furthermore, some conditions involve metabolic derangement, such as dyslipidemia, obesity, and diabetes, which have been shown to be possible risk factors for blepharoptosis $[1,6,13,14]$.

To the best of our knowledge, no study has assessed the association between MetS and blepharoptosis in the population, which is representative of the entire population, including young and elderly people. Therefore, in this study, we investigated the relationship between MetS and blepharoptosis in the Korea National Health and Nutrition Examination Survey (KNHANES) conducted in 2010 and 2012.

\section{Materials and methods}

\section{Study population}

This study was based on data from the 2010 to 2012 KNHANES. The KNHANES, conducted by the Korea Centers for Disease Control and Prevention, is a nationwide representative, cross-sectional, population-based survey used to assess the health and nutritional status of Koreans. These surveys have been conducted periodically since 1998 by the Korean Ministry of Health and Welfare by using a rolling sampling design, involving a complex, stratified, multistage, probability cluster survey of a representative sample of the non-institutionalized civilian population to assess the health and nutritional status of the Korean population $[15,16]$. The survey consists of the health interview, health examination, and nutrition surveys. This survey was reviewed and approved by the Institutional Review Board of the Korea Centers for Disease Control and Prevention, and all participants provided written informed consent.

In the present analysis, we limited the study population to adults aged 19 years or older who participated in all three parts of the survey in addition to ophthalmologic interviews and examinations. Our final study population for the analysis included 16,595 participants.

\section{Assessment of blepharoptosis}

Blepharoptosis was defined when the marginal reflex distance 1 (MRD1) was <2 mm $[13,14,17]$. MRD1 was measured as follows: After positioning at the eye level of the physician, each participant was instructed to look straight ahead and relax while focusing on a distant target. Using a penlight directed into the eye, the distance from the corneal light reflex to the upper eyelid margin was measured in millimeters [13]. The five stages of upper eyelid level (blepharoptosis severity) were defined as follows: stage $1=\mathrm{MRD} 1 \geq 4 \mathrm{~mm}$; stage $2=\mathrm{MRD} 13-3.9 \mathrm{~mm}$; stage 3=MRD1 2-2.9 mm; stage 4=MRD1 1-1.9 mm; and stage $5=\mathrm{MRD} 1<1 \mathrm{~mm}$ [12]. Subjects of stages 4 and 5 were considered to have blepharoptosis. All MRD1s were measured by specially trained ophthalmic resident physicians with $>3$ years of experience.

\section{Definition of MetS}

MetS was defined based on the Joint Interim Statement proposed in 2009 from the IDF and AHA/NHLBI [8]. Subjects were classified as having MetS if any three of the following five characteristics were present: (1) abdominal obesity (waist circumference $[\mathrm{WC}] \geq 90 \mathrm{~cm}$ in men or $\geq 80 \mathrm{~cm}$ in women, based on the IDF criteria), (2) fasting triglyceride (TG) level $\geq 150 \mathrm{mg} /$ $\mathrm{dl}$ or cholesterol-lowering medication use, (3) HDL cholesterol level $<40 \mathrm{mg} / \mathrm{dl}$ in men or $<50 \mathrm{mg} / \mathrm{dl}$ in women or cholesterollowering medication use, (4) systolic blood pressure $\geq 130$ $\mathrm{mmHg}$ and/or diastolic blood pressure $\geq 85 \mathrm{mmHg}$ or current use of medication for hypertension, and (5) fasting glucose $\geq 100$ $\mathrm{mg} / \mathrm{dl}$ or use of oral medication or insulin.

\section{Measurement}

The health interview survey was performed by trained interviewers. The demographic and socioeconomic characteristics, including education, income, smoking, drinking, and exercise, were obtained from the participants.

Anthropometric measurements of the participants were performed by specially trained examiners. WC was measured to 
the nearest $0.1 \mathrm{~cm}$ in a horizontal plane at the level of the midpoint between the iliac crest and the costal margin at the end of a normal expiration. The body mass index (BMI) was calculated as the individual's weight in kilograms divided by the square of the individual's height in meters. Blood pressure was measured three times on the right arm while the individual was in a seated position after at least 5 minutes of rest by using a mercury sphygmomanometer (Baumanometer; Baum, Copiague, NY, USA). The final blood pressure value was obtained by averaging the values of the second and third blood pressure measurements.

Blood samples were obtained after a minimum fasting time of 8 hours. Serum glucose, high-density lipoprotein (HDL) cholesterol, low-density lipoprotein (LDL) cholesterol, and TG levels were measured using the Hitachi automatic analyzer 7600 (Hitachi, Tokyo, Japan).

\section{Statistical analysis}

All data are presented as the mean \pm standard error (SE) for continuous variables or as proportions (with SEs) for categorical variables. Statistical analyses were conducted using the SAS (version 9.2; SAS Institute, Cary, NC, USA) survey procedure; this considered the complex sampling design (with sampling weights) of KNHANES and yielded nationally representative estimates of prevalence. We used two independent t-tests to compare continuous variables and the Rao-Scott chi-squared test to compare categorical variables. Multiple logistic regression analyses were performed to estimate the magnitude of the association between blepharoptosis and Mets. We used two statistical models, depending on the characteristics of the variables evaluated. The ORs and 95\% CIs were estimated after adjustment for potential confounders. Model 1 was adjusted for age, sex, and BMI. Model 2 was further adjusted for smoking, drinking, exercise, income, and education. $\mathrm{p}<0.05$ was consid-

Table 1. Characteristics of participants with or without blepharoptosis

\begin{tabular}{|c|c|c|c|}
\hline \multirow{2}{*}{ Characteristic } & \multicolumn{2}{|c|}{ Blepharoptosis } & \multirow{2}{*}{ p-value } \\
\hline & No $(n=14,684)$ & Yes $(n=1,911)$ & \\
\hline Age (yr) & $43.7 \pm 0.2$ & $59.2 \pm 0.7$ & $<0.0001$ \\
\hline BMI $\left(\mathrm{kg} / \mathrm{m}^{2}\right)$ & $23.6 \pm 0$ & $24.4 \pm 0.1$ & $<0.0001$ \\
\hline $\mathrm{WC}(\mathrm{cm})$ & $80.7 \pm 0.1$ & $84.6 \pm 0.3$ & $<0.0001$ \\
\hline SBP (mmHg) & $117.3 \pm 0.2$ & $125.6 \pm 0.6$ & $<0.0001$ \\
\hline DBP (mmHg) & $76.4 \pm 0.1$ & $77.1 \pm 0.3$ & 0.0808 \\
\hline Fasting glucose (mg/dl) & $95.8 \pm 0.2$ & $104.3 \pm 0.7$ & $<0.0001$ \\
\hline HDL-C (mg/dl) & $52.9 \pm 0.2$ & $49.4 \pm 0.4$ & $<0.0001$ \\
\hline LDL-C (mg/dl) & $109.9 \pm 0.4$ & $112.1 \pm 1.0$ & 0.038 \\
\hline $\mathrm{TG}(\mathrm{mg} / \mathrm{dl})^{\mathrm{a})}$ & $106.7(105.3-108.1)$ & $128.5(124.1-133.2)$ & $<0.0001$ \\
\hline $\mathrm{TC}(\mathrm{mg} / \mathrm{dl})$ & $187.9 \pm 0.4$ & $190.9 \pm 1.0$ & 0.0088 \\
\hline
\end{tabular}

Values are presented as mean \pm standard error for continuous variables.

Two independent $\mathrm{t}$ tests for quantitative data was performed.

BMI, body mass index; WC, waist circumference; SBP, systolic blood pressure; DBP, diastolic blood pressure; HDL-C, high-density lipoprotein cholesterol; LDL-C, low-density lipoprotein cholesterol; TG, triglyceride; TC, total cholesterol.

${ }^{a)}$ Geometric mean (95\% Confidence interval).

Table 2. Prevalence of metabolic syndrome (Mets) components according to upper eyelid level stage

\begin{tabular}{|c|c|c|c|c|c|c|}
\hline \multirow{2}{*}{ MetS component } & \multicolumn{5}{|c|}{ Upper eyelid level stage $^{\text {a) }}$} & \multirow{2}{*}{ p-value } \\
\hline & $\geq 4 \mathrm{~mm}$ & 3-3.9 mm & $2-2.9 \mathrm{~mm}$ & $1-1.9 \mathrm{~mm}$ & $<1 \mathrm{~mm}$ & \\
\hline $\mathrm{WC}(\geq 90$ or $\geq 80 \mathrm{~cm})$ & $26.5(0.8)$ & $32.3(1.0)$ & $38.5(1.4)$ & $46.5(1.8)$ & $53.5(3.3)$ & $<0.0001$ \\
\hline Hypertension & $26.8(0.8)$ & $37.6(1.0)$ & $46.5(1.5)$ & $57.0(1.9)$ & $67.0(3.0)$ & $<0.0001$ \\
\hline Hyperglycemia & $19.0(0.7)$ & $26.4(0.9)$ & $31.8(1.2)$ & $41.6(1.9)$ & $43.9(3.0)$ & $<0.0001$ \\
\hline Hypertriglyceridemia & $26.3(0.8)$ & $32.0(0.8)$ & $36.0(1.1)$ & $44.1(2.0)$ & $45.7(3.1)$ & $<0.0001$ \\
\hline Low HDL-C & $25.2(0.7)$ & $30.1(0.8)$ & $34.1(1.0)$ & $40.4(1.8)$ & $45.0(2.7)$ & $<0.0001$ \\
\hline MetS & $18.1(0.6)$ & $26.5(0.8)$ & $33.4(1.2)$ & $45.6(1.7)$ & $54.0(3.1)$ & $<0.0001$ \\
\hline
\end{tabular}

Values are presented as weighted \% (standard error).

Rao-Scott chi-square test was performed.

MetS, metabolic syndrome; WC, waist circumference; HDL-C, high-density lipoprotein cholesterol.

${ }^{a)}$ By marginal reflex distance 1 . 
ered statistically significant.

The study was approved by the Institutional Review Board at The Catholic University of Korea and adhered to the tenets of the Declaration of Helsinki.

\section{Results}

Table 1 shows the characteristics of the participants, including MetS components by blepharoptosis status. Compared with subjects without blepharoptosis, those with blepharoptosis had higher mean age, BMI, WC, systolic blood pressure, fasting glucose, total cholesterol (TC), LDL cholesterol, and TG; meanwhile, they had lower HDL cholesterol.

Table 2 shows the prevalence of MetS components in accordance with upper eyelid level stage. As the upper eyelid level stage increased, the prevalence of MetS and its components increased.

Fig. 1 shows the prevalence of blepharoptosis based on the number of MetS components. As the number of MetS components increased, the prevalence of blepharoptosis also in-

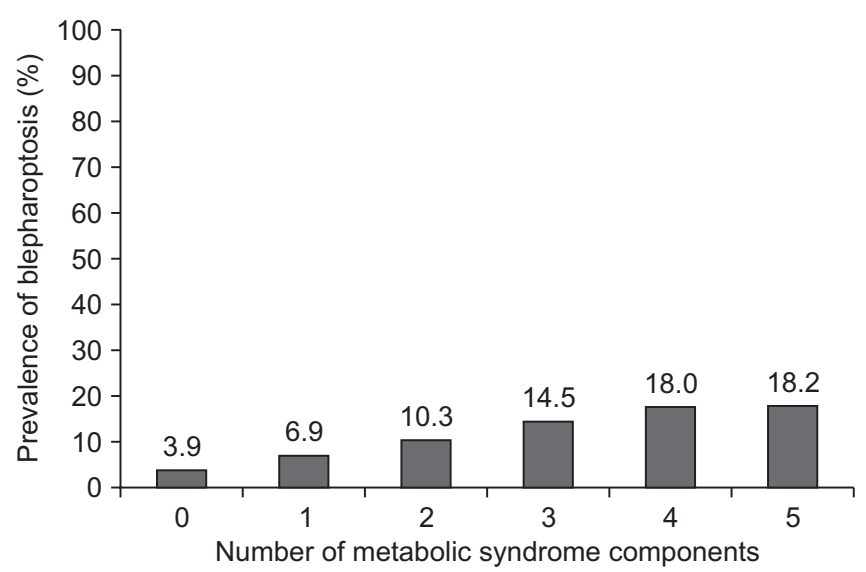

Fig. 1. Prevalence of blepharoptosis based on the number of metabolic syndrome components $(\mathrm{p}<0.0001$ by chi-square test). creased.

The adjusted ORs (aOR) and their 95\% CIs of MetS components for blepharoptosis are shown in Table 3. We used three models with progressive degrees of adjustment. Model 1 was not adjusted. Model 2 was adjusted for age, sex, and BMI. Model 3 was further adjusted for smoking, drinking, exercise, income, and education. After being controlled for confounders, MetS was significantly associated with blepharoptosis (aOR, 1.316; 95\% CI, 1.133-1.529). Increased WC, hypertension, and elevated TGs were also significantly associated with blepharoptosis (aOR, 95\% CI; 1.281 (1.047-1.567), 1.165 (1.014-1.339), and 1.174 (1.013-1.36), respectively).

\section{Discussion}

This study investigated whether MetS and its components are associated with blepharoptosis in Korean adults. We found that MetS and its components showed a strong association with blepharoptosis in the Korean population. The positive association of MetS with blepharoptosis remained significant even after adjusting for confounding factors (Table 3). Interestingly, as the number of MetS components increased, the prevalence of blepharoptosis also increased (Fig. $1 ; \mathrm{p}<0.0001$ ). As the upper eyelid level stage increased, the prevalence of MetS and its components increased (Table 2).

Both MetS and blepharoptosis are important health issues. Diabetes and insulin-resistant state, such as impaired glucose tolerance, are also known to be associated with blepharoptosis [5]. A recent study showed that diabetes is an independent risk factor for blepharoptosis in the general Korean population [6]. However, to the best of our knowledge, no study has assessed the association of MetS with blepharoptosis in the population, which is representative of the entire population, including young and elderly people. Interestingly, increased WC, hypertension, and elevated TG, as well as MetS, also increased the

Table 3. Odds ratios (95\% confidence intervals) for blepharoptosis according to components of MetS

\begin{tabular}{lccc}
\hline \multicolumn{1}{c}{ MetS components } & Model 1 $^{\text {a) }}$ & Model 2 $^{\text {b) }}$ & Model 3 $^{\text {c) }}$ \\
\hline WC $(\geq 90$ or $\geq 80 \mathrm{~cm})$ & $2.01(1.779,2.271)$ & $1.299(1.07,1.578)$ & $1.281(1.047,1.567)$ \\
Hypertension & $2.81(2.46,3.21)$ & $1.146(1,1.313)$ & $1.165(1.014,1.339)$ \\
Hyperglycemia & $2.278(2.005,2.59)$ & $1.14(0.992,1.309)$ & $1.144(0.992,1.319)$ \\
Hypertriglyceridemia & $1.838(1.597,2.116)$ & $1.198(1.034,1.389)$ & $1.174(1.013,1.36)$ \\
Low HDL-C & $1.708(1.498,1.947)$ & $1.117(0.958,1.303)$ & $1.078(0.927,1.253)$ \\
MetS & $2.783(2.476,3.128)$ & $1.343(1.164,1.55)$ & $1.316(1.133,1.529)$ \\
\hline
\end{tabular}

Multiple logistic regression analysis was performed.

MetS, metabolic syndrome; WC, waist circumference; HDL-C, high-density lipoprotein cholesterol.

${ }^{a}$ U) Unadjusted, ${ }^{\text {b) }}$ adjusted for age, sex, and body mass index (BMI), ${ }^{\text {c) }}$ adjusted for age, sex, BMI, smoking, drinking, exercise, income, and education. 
prevalence of blepharoptosis.

Blepharoptosis is defined as abnormally low-lying upper eyelid margin in primary gaze, resulting in narrowing of the palpebral opening and fissure and covering part of the eye. The normal adult upper lid margin is $0.5-2 \mathrm{~mm}$ below the superior corneal limbus and is highest just nasal to the pupil (upper lid height) $[18,19]$. The deficient function may be due to weakness of the muscle responsible for lid retraction (either the levator palpebrae superioris muscle and its aponeurosis or Muller's muscle), or damage to the nerves that control these muscles [1]. The prevalence of blepharoptosis in Korean adults older than 19 years was $11.5 \%$, which was similar to the result of the previous study at $11.0 \pm 0.8 \%$ [13].

The prevalence of the MetS, a cluster of cardiovascular risk factors associated with obesity and insulin resistance, is dramatically increasing in Western and developing countries. This disorder consists of metabolic conditions, such as hypertriglyceridemia, hyper-LDL cholesterol, hypo-HDL cholesterol, insulin resistance, abnormal glucose tolerance, and hypertension, that-in combination with genetic susceptibility and abdominal obesity are risk factors for type 2 diabetes, vascular inflammation, atherosclerosis, and renal, liver and heart diseases [20]. One of the defects in MetS and its associated diseases is excess of reactive oxygen species. Reactive oxygen species generated by the mitochondria, or from other sites within or outside the cell, cause damage to mitochondrial components and initiate degradative processes. Such toxic reactions contribute significantly to the aging process [21]. In this study, the patients with MetS were more likely to be older compared with the participants who lacked MetS (Table 1, p<0.0001).

We have established some hypotheses regarding the association of MetS and its components with blepharoptosis. First, increased reactive oxygen species resulting from MetS may cause damage to the nerve that control the levator palpebrae superioris muscle. Second, it is highly possible that blepharoptosis patients who have MetS are obese and have diffuse fatty infiltration in the levator palpebrae superioris muscle and its aponeurosis.

In conclusion, in this nationally representative populationbased cross-sectional study, we found that MetS was positively associated with blepharoptosis in Korean adults. Especially, the more patients had the number of MetS components, the more severe blepharoptosis digress was. Therefore, screening for blepharoptosis is warranted for patients with MetS.

\section{Conflicts of interest}

The authors have nothing to disclose.

\section{References}

1. Finsterer J. Ptosis: causes, presentation, and management. Aesthetic Plast Surg 2003;27:193-204.

2. Clauser L, Tieghi R, Galiè M. Palpebral ptosis: clinical classification, differential diagnosis, and surgical guidelines: an overview. J Craniofac Surg 2006;17:246-54.

3. Beard C. A new classification of blepharoptosis. Int Ophthalmol Clin 1989;29:214-6.

4. de Figueiredo AR. Blepharoptosis. Semin Ophthalmol 2010; 25:39-51.

5. Bosco D, Costa R, Plastino M, Branca D, Cotronei P, Sperlì T, et al. Glucose metabolism in the idiopathic blepharoptosis: utility of the oral glucose tolerance test (OGTT) and of the insulin resistance index. J Neurol Sci 2009;284:24-8.

6. Moon SS, Lee YS. Prevalence and clinical characteristics of blepharoptosis in patients with diabetes in the Korea National Health and Nutrition Examination Survey (KNHANES) 20092010. Endocrine 2015;48:504-10.

7. Chen WL, Wang CC, Wu LW, Kao TW, Chan JY, Chen YJ, et al. Relationship between lung function and metabolic syndrome. PLoS One 2014;9:e108989.

8. Alberti KG, Eckel RH, Grundy SM, Zimmet PZ, Cleeman JI, Donato KA, et al.; International Diabetes Federation Task Force on Epidemiology and Prevention; Hational Heart, Lung, and Blood Institute; American Heart Association; World Heart Federation; International Atherosclerosis Society; International Association for the Study of Obesity. Harmonizing the metabolic syndrome: a joint interim statement of the International Diabetes Federation Task Force on Epidemiology and Prevention; National Heart, Lung, and Blood Institute; American Heart Association; World Heart Federation; International Atherosclerosis Society; and International Association for the Study of Obesity. Circulation. 2009;120:1640-5.

9. Sumner CJ, Sheth S, Griffin JW, Cornblath DR, Polydefkis M. The spectrum of neuropathy in diabetes and impaired glucose tolerance. Neurology 2003;60:108-11.

10. Gordon Smith A, Robinson Singleton J. Idiopathic neuropathy, prediabetes and the metabolic syndrome. J Neurol Sci 2006;242:9-14.

11. Smith AG, Singleton JR. Impaired glucose tolerance and neuropathy. Neurologist 2008;14:23-9. 
12. Paik JS, Jung SK, Han KD, Kim SD, Park YM, Yang SW. Obesity as a potential risk factor for blepharoptosis: the Korea National Health and Nutrition Examination Survey 2008-2010. PLoS One 2015;10:e0131427.

13. Yoon KC, Mun GH, Kim SD, Kim SH, Kim CY, Park KH, et al. Prevalence of eye diseases in South Korea: data from the Korea National Health and Nutrition Examination Survey 20082009. Korean J Ophthalmol 2011;25:421-33.

14. Shirado M. Dyslipidaemia and age-related involutional blepharoptosis. J Plast Reconstr Aesthet Surg 2012;65:e146-50.

15. Moon SS. Association of lead, mercury and cadmium with diabetes in the Korean population: the Korea National Health and Nutrition Examination Survey (KNHANES) 2009-2010. Diabet Med 2013;30:e143-8.

16. Lee J, Lee S, Jang S, Ryu OH. Age-related changes in the prevalence of osteoporosis according to gender and skeletal site: the Korea National Health and Nutrition Examination Survey 2008-2010. Endocrinol Metab (Seoul) 2013;28:180-91.

17. Small RG, Sabates NR, Burrows D. The measurement and definition of ptosis. Ophthalmic Plast Reconstr Surg 1989;5:171-5.

18. Iliff JW, Pacheco EM. Ptosis surgery. In: Duane TD, Tasman W, Jaeger EA, editors. Duane's clinical ophthalmology on CDROM. Philadelphia, PA: Lippincott Williams and Wilkins; 2001. p. 1-18.

19. Black E, Nesi FA, Calvano CJ, Gladstone GJ, Levine MR. Smith and Nesi's ophthalmic plastic and reconstructive surgery. 3rd ed. New York: Springer; 2012.

20. Bonomini F, Rodella LF, Rezzani R. Metabolic syndrome, aging and involvement of oxidative stress. Aging Dis 2015;6:10920.

21. Harman D. Aging: a theory based on free radical and radiation chemistry. J Gerontol 1956;11:298-300. 